\title{
COMPARISON OF AXIAL FAN ROTOR EXPERIMENTAL DATA WITH CFD SIMULATION
}

\begin{abstract}
Alě̌ PRACHAř
Aerospace Research and Test Establishment, Beranových 130, 19905 Praha-Letñany, Czech Republic correspondence: prachar@vzlu.cz

Abstract. Data obtained from an experimental simulation on a new test rig for axial fans are compared to a CFD simulation. The Edge solver is used and the development needed for the simulation (boundary conditions, free stream consistency) is described. Adequate agreement between the measured and calculated data is observed.
\end{abstract}

KEYworDs: axial fan; CFD; Edge software; boundary conditions.

AMS Mathematics Subject Classification: 65N08, 76M12.

\section{INTRODUCTION}

Axial flow fans are used in many applications ranging from cooling of the computer CPU's to the propulsion of wind tunnels or cooling towers of the power plants, to emphasize extreme scales.

For better understanding of the flow phenomena it is usual and today almost inevitable to include CFD into the design and testing. It is, however, necessary to develop sufficiently accurate computational tools and validate them extensively.

A test rig for axial fans has been designed and constructed in recent years. Compared to the previous state it allows automatic flow regulation and data acquisition. From the point of view of the CFD user it serves as a valuable source of validation data for computational methods.

The Edge CFD solver package [1] has been used so far mainly for external aerodynamics problems with encouraging results 2 . Since the solver includes many of the ingredients needed to solve problems in rotating domains (turbomachinery), it was decided to test its ability to deal with this kind of problems. Several updates, corrections and generalizations of the existing code had to be developed for the required functionality.

This paper starts with a brief description of the experimental device and the data obtained. However, the main part is dedicated to the development of the Edge flow solver and to a comparison with the experimental data.

\section{EXPERIMENTAL SET-UP AND MEASURED DATA}

A test rig for the axial fans, see Fig. 1, has been designed. Since the electric motor has been placed upstream of the fan rotor and the shaft is relatively short to prevent problems with vibrations, the inlet channel has been designed to change the direction of the incoming air from radial (centripetal) to axial.
The shaft is fitted with dynamometer to measure torque.

The hub and the shroud (casing) are fixed together by the struts which are placed upstream (airfoil shape) and downstream of the rotor. The diameter of the shroud at the rotor position is $630 \mathrm{~mm}$ and the hub to shroud diameter ratio is 0.55 .

To vary the volume flow rate by aerodynamic resistance, the adjustable choking element is mounted at the outflow area.

The properties of the flow (total and static pressure, velocity vector) are measured at the planes approximately $130 \mathrm{~mm}$ upstream and downstream of the fan rotor by a pair of 5-hole pitot-static probes (see Fig. 1) traversing along the radial direction. The test rig has a constant cross section between those two planes.

The measured quantities, obtained as functions of radial coordinate, were later utilized to calculate integral values (volume flow rate, average total or static pressure).

\section{CFD Simulation}

For the CFD simulation the Edge software, see [3], which is a compressible flow solver for unstructured grids with arbitrary elements was used. Edge is based on the node-centred finite volume formulation of the Euler or the Navier-Stokes equations in two or three dimensions using dual grids.

\subsection{SOLVER DESCRIPTION}

The solver has been used in its parallel version utilizing convergence acceleration techniques like multigrid, low speed preconditioning [4], implicit residual smoothing and local time stepping.

The flow equations formulated in a reference frame rotating around an arbitrary axis $\Omega$ with an angular velocity $|\Omega|$ are written with the aid of the Einstein summation convention as

$$
\frac{\partial U}{\partial t}+\frac{\partial F_{i}}{\partial x_{i}}+\frac{\partial G_{i}}{\partial x_{i}}=S
$$




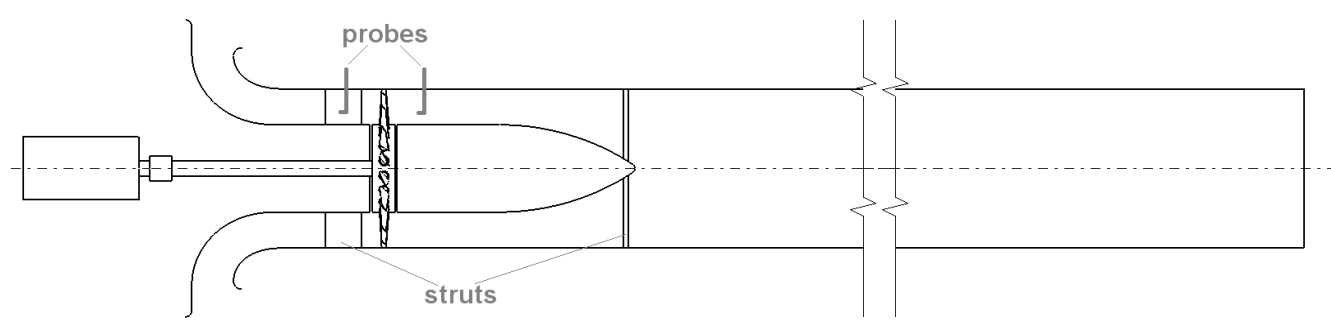

Figure 1. Test stand scheme. A pair of 5-hole pitot-static probes is placed upstream and downstream of the fan rotor.

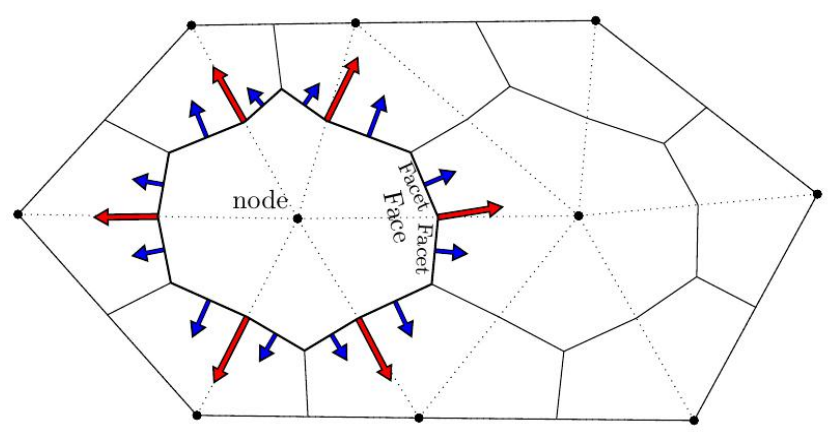

Figure 2. Primary (dotted) and dual grid (solid), the vectors representing facets (blue) and faces (red).

The definition of unknowns $U$, convective fluxes $F_{i}$ and the source term $S$ is given by

$$
\begin{gathered}
U=\left(\begin{array}{c}
\rho \\
\rho u_{1} \\
\rho u_{2} \\
\rho u_{3} \\
\rho E_{r}
\end{array}\right), \quad F_{i}=\left(\begin{array}{c}
\rho w_{i} \\
\rho u_{1} w_{i}+\delta_{i 1} p \\
\rho u_{2} w_{i}+\delta_{i 2} p \\
\rho u_{3} w_{i}+\delta_{i 3} p \\
\left(\rho E_{r}+p\right) w_{i}
\end{array}\right), \\
S=\left(\begin{array}{c}
0 \\
\rho \Omega \times u \\
0
\end{array}\right),
\end{gathered}
$$

where $u$ denotes the absolute velocity vector and $w$ stands for the relative velocity. The exact form of the viscous fluxes $G_{i}$ is omitted here for brevity. Let us note that the total energy in the energy equation contains contribution from the rotation

$$
E_{r}=E-u \cdot(\Omega \times r) .
$$

Since the grid movement (rotation) is included in the governing equations, they can be solved in a steady state manner.

For our simulation various $k-\omega$ models of turbulence have been tested, and the low Reynolds number model [5] has been used for the presented results.

In the Finite volume method, (1) is solved in the integral form

$$
\frac{d}{d t} \int_{\mathcal{V}} U+\int_{\partial \mathcal{V}}\left(F_{i}+G_{i}\right) \cdot n=\int_{\mathcal{V}} S
$$

For the convective fluxes the second order central scheme with the Jameson-Schmidt-Turkel type artificial dissipation term has been used.
The data structure for the finite volume flux calculations is edge-based and the reduced scheme is used, i.e., the normals representing the facets between two adjacent dual cells are summed into a single one, which is kept in the data structure, see Fig. 2. Hence, only one flux evaluation between two adjacent cells is needed.

It was noted by Raichle [6] that discretization error is introduced if the grid velocity $\Omega \times r$ at the cell face is averaged from the cell centres. He proposed a formulation assuring exact integration and, hence, free stream consistency for rotating flows. The scheme, still based on reduced formulation, was implemented.

The only additional requirement is that along with the computed normals, representing the cell faces, additional vector for each face has to be stored in the data structure. For a typical case we observed increased memory demands for up to 10-15\% compared to the inexact reduced scheme. Increase in the CPU time is negligible.

\subsection{BOUNDARY CONDITIONS}

For internal aerodynamics it is usually natural to use the pair of boundary conditions prescribing total quantities (total pressure, total temperature and flow direction) at the inlet and the static pressure at the outlet from the computational domain. We consider the inlet boundary condition adequate for our case.

However, the exit static pressure is influenced by the performance of the rotor at various volume flow rates. If we inspect the dependence of the exit static pressure on the volume flow rate, see Fig. 3, we find that the static pressure alone does not uniquely determine the flow regime. We prescribe the average of boundary 


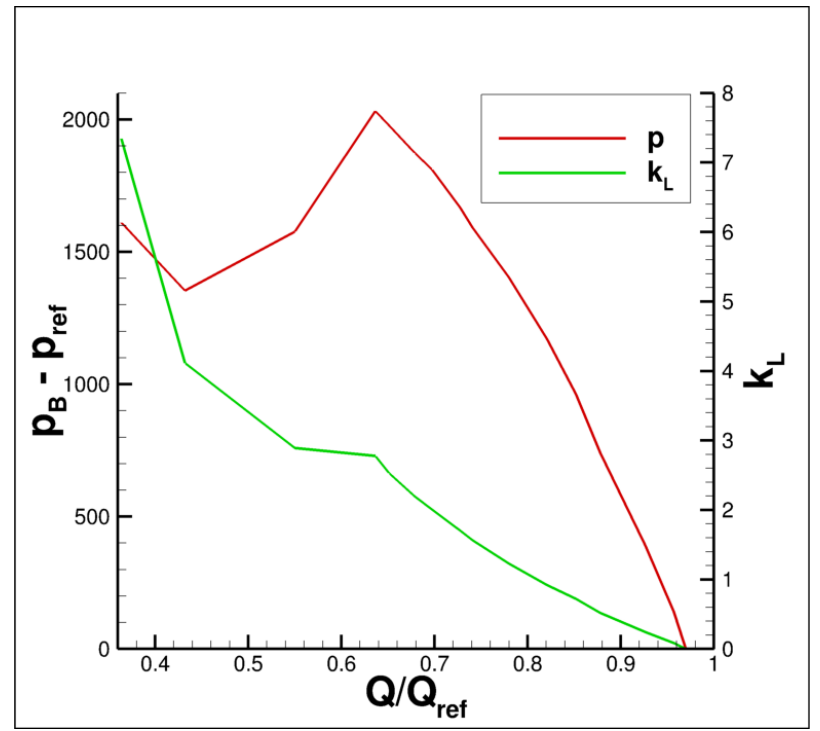

FiguRE 3. Outlet static pressure (relative) and corresponding $k_{L}$ parameter for a typical case.

pressure $\tilde{p}_{b}$ according to

$$
\tilde{p}_{b}=p_{\text {ref }}+\frac{1}{2} k_{L} \tilde{\rho} \tilde{u}_{a x}^{2},
$$

where $p_{\text {ref }}$ denotes reference pressure and $k_{L}$ is a free parameter. The calculation was carried out for a range of $k_{L}$ 's to cover various flow regimes.

Moreover, since the flow exhibits large circumferential (peripheral) velocities as a consequence of the absence of stator blades, the outlet boundary condition is implemented to satisfy the radial equilibrium condition which we assume at the outlet and which is considered in a simple form, cf. [7,

$$
\frac{d p_{b}}{d r}=\frac{\rho u_{\theta}^{2}}{r} .
$$

Finally, $p_{b}$ is updated to vary along the radial coordinate taking into account both (5) and (6).

Another type of boundary used in our simulation is a solid wall. However, we need to distinguish between boundaries steady with respect to the fixed coordinates (e.g., casing) where $u=0$ is prescribed, and boundaries moving with the reference frame (e.g., rotor blade) with $w=0$. In Edge, weak formulations for these boundary conditions are used, 8 .

Since no information about the turbulence level was known, the inlet turbulence intensity was set to $1 \%$ of the inlet velocity and the freestream viscosity ratio (turbulent to dynamic viscosity) was set to the default value equal to 1 .

\subsection{CFD Geometry}

For the CFD calculation the model was simplified considerably. First, the struts were removed and only the rotor blades were preserved. This makes the problem rotationally periodic. Only one rotor blade was modelled and the rotation periodic boundary condition

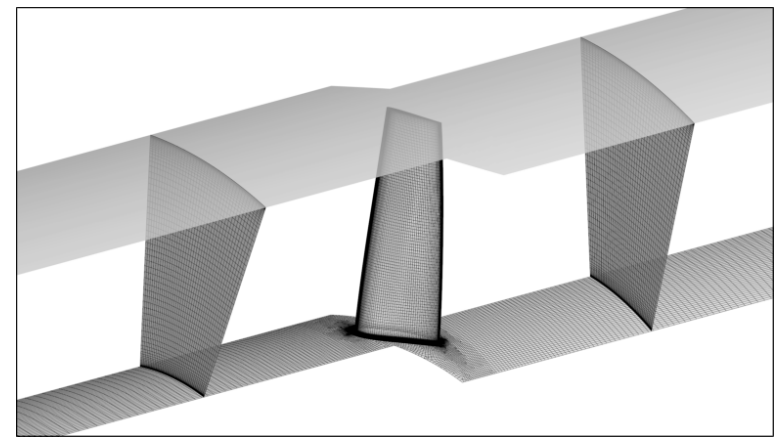

Figure 4. Layout of the CFD simulation.

was applied. The CFD geometry models the constant cross-section part of the test rig, prolonged upstream and downstream.

A hybrid (tetrahedral and hexahedral elements) computational grid was used as a primary grid with approximately 2.3 million nodes. The layout of the simulation and of the planes where the flow properties were evaluated is indicated in Fig. 4 The structured hexahedral blocks were placed upstream and downstream of the rotor. The structured block with O-grid topology was used around the rotor blade with 100 points on each side of the blade surface, 35 points in the normal direction and 120 radial grid points. The boundary layer was fully resolved around the solid walls and in the tip gap to keep the parameter $\mathrm{y}+$ below 1. The cell expansion ratio from the wall was kept close to 1.2. The rest of the space between the blade O-grid block and the periodic boundaries was filled with tetrahedral elements.

\section{Comparison of EXPERIMENT DATA WITH CFD RESULTS}

To match the experimental data the flow field obtained from the CFD simulation was evaluated at the planes upstream and downstream of the rotor. The volume flow rates, average of static and total pressure are the basic data which together with torque at the rotor blades are used to calculate integral values.

The experimental data were obtained for three settings of blade angle $\left(25^{\circ}, 35^{\circ}\right.$ and $\left.45^{\circ}\right)$, constant angular velocity $\left(|\Omega|=394 \mathrm{rad} / \mathrm{s}, u_{t}=124 \mathrm{~m} / \mathrm{s}\right)$ and for a range of choking element settings from fully open to and beyond the blade stall. The non-dimensional pressure and flow coefficients are used for comparison, see Fig. 5 The notation is similar to 9 .

We observe satisfactory agreement of experimental and CFD data. Let us note that the computation was performed for several settings of the solver. The choice of turbulence model had a notable effect on the prediction of the blade stall. In our cases the low Reynolds numbers $k-\omega$ turbulence model [5] gave the best results in terms of capturing the blade stall. The cases in the stall region for the given blade settings are averaged values since oscillatory behaviour was 


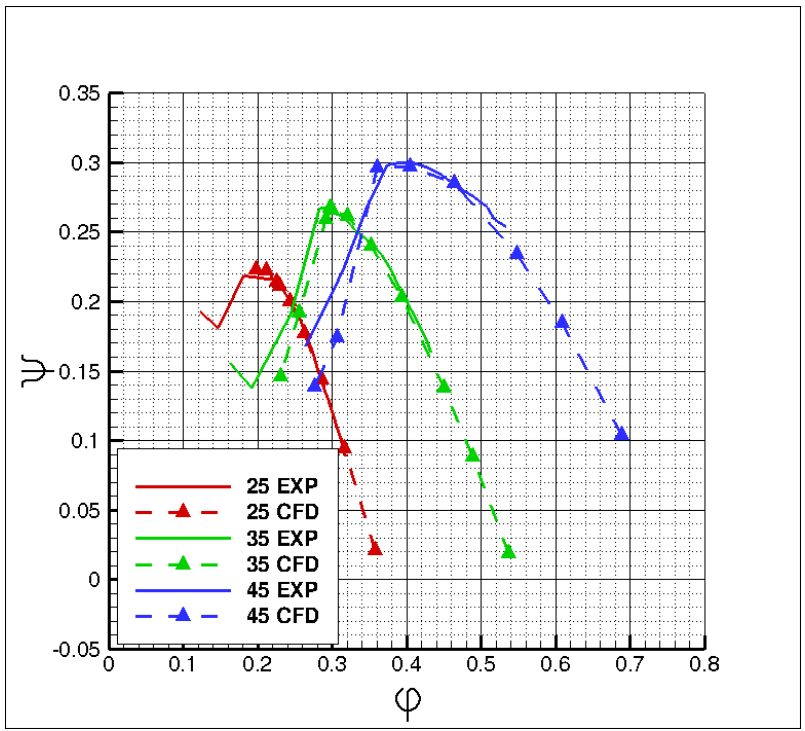

FiguRE 5. Axial fan performance in non-dimensional parameters for various blade angles. Full line denotes the experiment, dashed lines with triangles represent CFD calculation.

observed. In this case the unsteady solution could be used to improve accuracy.

Extensive validation of the turbulence models is necessary for the class of problems considered in this paper since testing of their implementation in Edge was done mainly for external flows. Various other simplifications and idealizations can also cause differences, e.g., tip clearance height estimate, which is never perfectly uniform in the experiment, the effect of struts, etc.

The (relative) isentropic efficiency for the considered cases is shown in Fig. 6.

We can also consider the agreement of the data acceptable. The slightly higher efficiency for higher volume flow rates (especially for the blade setting of $35^{\circ}$ ) can be partly explained by the turbulence modelling. The flow is considered fully turbulent in CFD, however, turbulent transition could cause improvement.

\section{Conclusion}

The main result of this paper is that it is possible to use the Edge software, a code primarily designed for external aerodynamics, for the problem under consideration. Various enhancements were necessary for appropriate functionality. However, the changes to the code were compatible with the solver structure.

An acceptable agreement between the measured and calculated data was observed. Local quantities could be compared in the next step.

Future research will focus on the rotor-stator configuration and the modelling of the interface between the blade rows.

\section{LIST OF SYMBOLS}

$A$ Flow area (cross-section) $\left[\mathrm{m}^{2}\right]$

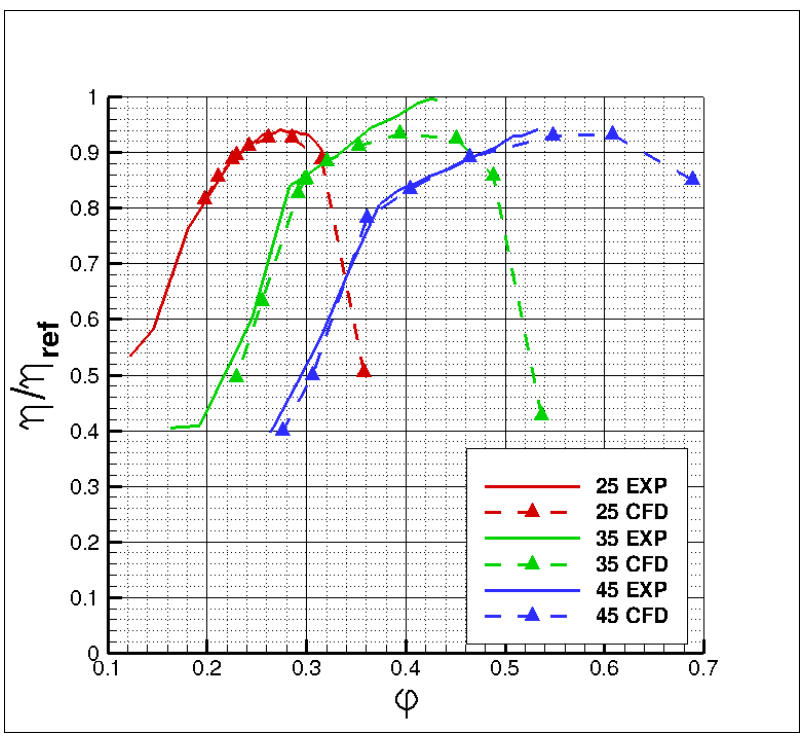

FiguRE 6. Relative efficiency for three blade settings. Full line denotes the experiment, dashed lines with triangles represent CFD calculation.

$M_{k}$ Torque moment [ $\left.\mathrm{Nm}\right]$

$p \quad$ Fluid pressure $[\mathrm{Pa}]$

$\tilde{p}_{T} \quad$ Total pressure (ISO), $\tilde{p}_{T}=\tilde{p}+\frac{1}{2} \tilde{\rho}\left(\frac{Q}{A}\right)^{2} \quad[\mathrm{~Pa}]$

$Q$ Volume flow rate $\left[\mathrm{m}^{3} \mathrm{~s}^{-1}\right]$

$r$ Radial coordinate $[\mathrm{m}]$

$u$ Absolute velocity vector $\left[\mathrm{m} \mathrm{s}^{-1}\right]$

$u_{a x}$ Axial component of velocity $\left[\mathrm{m} \mathrm{s}^{-1}\right]$

$u_{\theta} \quad$ Peripheral component of velocity $\left[\mathrm{m} \mathrm{s}^{-1}\right]$

$u_{t} \quad$ Blade tip velocity $\left[\mathrm{m} \mathrm{s}^{-1}\right]$

$w \quad$ Relative velocity vector, $w=u-\Omega \times r\left[\mathrm{~m} \mathrm{~s}^{-1}\right]$

$\delta_{i j} \quad$ Kronecker delta

$\eta \quad$ Isentropic efficiency $\quad \eta=Q \Delta \tilde{p}_{T} /\left(M_{k}|\Omega|\right)$

$\rho \quad$ Fluid density $\left[\mathrm{kg} \mathrm{m}^{-3}\right]$

$\varphi$ Flow coefficient $\varphi=Q /\left(A u_{t}\right)$

$\psi \quad$ Pressure coefficient $\quad \psi=2 \Delta \tilde{p}_{T} /\left(\tilde{\rho} u_{t}^{2}\right)$

$\Omega$ Rotation axis, angular velocity $|\Omega|[\mathrm{rad} / \mathrm{s}]$

( ) Average of the quantity across boundary

()$_{b}$ Boundary value

\section{ACKNOWLEDGEMENTS}

This research was supported by The Ministry of Industry and Trade of the Czech Republic for long-term strategic development. Access to computing and storage facilities owned by parties and projects contributing to the $\mathrm{Na}$ tional Grid Infrastructure MetaCentrum, provided under the "Projects of Large Infrastructure for Research, Development, and Innovations" programme (LM2010005), is greatly appreciated.

\section{REFERENCES}

[1] P. Eliasson. Edge, a Navier-Stokes solver for unstructured grids. In Proceedings to Finite Volumes for Complex Applications III., pp. 527-534. ISTE Ltd., London, 2002.

[2] P. Eliasson, S.-H. Peng, L. Tysell. Computations from the fourth drag prediction workshop using the Edge 
solver. Journal of Aircraft 50(5):1646-1655, 2013. DOI:10.2514/1.C032225

[3] Edge theoretical formulation. Tech. Rep. 03-2870, Swedish Defence Research Agency (FOI), 2007.

[4] A. Prachař. Local low speed preconditioning in rotating reference frame. Applied Mathematical Sciences 9(5):209-218, 2015. DOI:10.12988/ams.2015.411885

[5] S.-H. Peng, L. Davidson, S. Holmberg. A modified low-Reynolds-number $k-\omega$ model for recirculating flows. ASME J Fluids Eng 119:867-875, 1997. DOI:10.1115/1.2819510

[6] A. Raichle. Extension of the unstructured TAU-code for rotating flows. In New Results in Numerical and Experimental Fluid Mechanics V, vol. 92 of Notes on Numerical Fluid Mechanics and Multidisciplinary Design (NNFM), pp. 136-143. Springer Berlin Heidelberg, 2006. DOI:10.1007/978-3-540-33287-9_17.
[7] D. L. Tweedt, R. V. Chima, E. Turkel.

Preconditioning for numerical simulation of low mach number three-dimensional viscous turbomachinery flows. In Proceedings of 28th Fluid Dynamics Conference. 1997. DOI:10.2514/6.1997-1828.

[8] P. Eliasson, S. Eriksson, J. Nordström. The influence of weak and strong solid wall boundary conditions on the convergence to steady-state of the navier-stokes equations. In Proceedings of 19th AIAA CFD Conferenc. 2009. DOI:10.2514/6.2009-3551.

[9] V. Cyrus, J. Cyrus, P. Wurst, P. Panek. Aerodynamic performance of advanced axial flow fan for power industry within its operational range. In Proceedings of ASME Turbo Expo. 2014. DOI:10.1115/GT2014-25339 